\title{
Association analysis of Notch pathway signalling genes in diabetic nephropathy
}

\author{
D. Kavanagh • G. J. McKay • C. C. Patterson • \\ A. J. McKnight • A. P. Maxwell • D. A. Savage • \\ the Warren 3/UK GoKinD Study Group
}

Received: 16 September 2010 / Accepted: 22 October 2010 /Published online: 20 November 2010

(C) Springer-Verlag 2010

\begin{abstract}
Aims/hypothesis Several studies have provided compelling evidence implicating the Notch signalling pathway in diabetic nephropathy. Co-regulation of Notch signalling pathway genes with GREM1 has recently been demonstrated and several genes involved in the Notch pathway are differentially expressed in kidney biopsies from individuals with diabetic nephropathy. We assessed single-nucleotide polymorphisms (SNPs; $n=42$ ) in four of these key genes (JAG1, HES1, NOTCH3 and ADAM10) for association with diabetic nephropathy using a case-control design.

Methods Tag SNPs and potentially functional SNPs were genotyped using Sequenom or Taqman technologies in a total of 1371 individuals with type 1 diabetes (668 patients with nephropathy and 703 controls without nephropathy). Patients and controls were white and recruited from the UK and Ireland. Association analyses were performed using
\end{abstract}

D. Kavanagh and G. J. McKay contributed equally to this study.

Electronic supplementary material The online version of this article (doi:10.1007/s00125-010-1978-3) contains supplementary material, which is available to authorised users.

D. Kavanagh • G. J. McKay • A. J. McKnight • A. P. Maxwell •

D. A. Savage $(\square)$

Nephrology Research Group, Centre for Public Health,

Queen's University Belfast,

Belfast, UK

e-mail: David.Savage@belfasttrust.hscni.net

C. C. Patterson

Epidemiology Research Group, Centre for Public Health, Queen's University Belfast,

Belfast, UK

\section{A. Savage}

Histocompatibility \& Immunogenetics Laboratory,

Blood Transfusion Building, Belfast City Hospital,

Lisburn Road,

Belfast BT9 7TS, UK
PLINK (http://pngu.mgh.harvard.edu/ purcell/plink/) and haplotype frequencies in patients and controls were compared. Adjustment for multiple testing was performed by permutation testing.

Results In analyses stratified by centre, we identified six SNPs, rs8708 and rs11699674 (JAG1), rs10423702 and rs1548555 (NOTCH3), rs2054096 and rs8027998 (ADAM10) as being associated with diabetic nephropathy before, but not after, adjustment for multiple testing. Haplotype and subgroup analysis according to duration of diabetes also failed to find an association with diabetic nephropathy.

Conclusions/interpretation Our results suggest that common variants in JAG1, HES1, NOTCH3 and ADAM10 are not strongly associated with diabetic nephropathy in type 1 diabetes among white individuals. Our findings, however, cannot entirely exclude these genes from involvement in the pathogenesis of diabetic nephropathy.

Keywords $A D A M 10$ - Diabetic nephropathy - Epithelial-tomesenchymal cell transition · Gremlin - HES1 - JAG1 . $\mathrm{NOTCH} 3 \cdot$ Notch signalling pathway
Abbreviations
DN Diabetic nephropathy
HWE Hardy-Weinberg equilibrium
MAF Minor allele frequency
NICD Notch intracellular domain
SNP Single-nucleotide polymorphism

\section{Introduction}

Recent studies have provided convincing evidence implicating members of the Notch signalling pathway in the pathogenesis of diabetic nephropathy (DN) [1-3]. Notch 
signalling is initiated following engagement of Notch transmembrane receptors by their ligands under the influence of several modifiers and transcription factors which, upon binding, result in cleavage of the extracellular Notch domain by a disintegrin and metallopeptidase domain 10 (ADAM10), followed by cleavage of the Notch intracellular domain (NICD) by $\gamma$-secretase [4]. The NICD translocates from the plasma membrane to the nucleus, where it associates with DNA binding proteins to regulate the transcription of target genes, including the HES and HEY family of helix-loop-helix proteins [4].

TGF $\beta 1$-induced epithelial-to-mesenchymal cell transition plays a key role in renal fibrosis [5], a characteristic of DN. Morrisey and colleagues [1] reported TGF $\beta 1$-mediated upregulation of Jagged 1 (a ligand of Notch) in kidney tubules in a mouse model of renal fibrosis. The developmentally regulated gene GREM1 encodes gremlin, which is a bone morphogenetic protein antagonist, and is implicated in the development of DN [6]. A recent study by Walsh and colleagues [2] revealed that the JAG1 and HES1 genes share common regulatory elements with GREM1. The authors observed upregulation of GREM1, JAG1 and HES1 in kidney epithelial cells exposed to TGF $\beta 1$. Furthermore, increased expression of these genes was demonstrated by quantitative RT-PCR in kidney biopsies from patients with DN compared with control biopsy samples [2]. In situ hybridisation studies of kidney biopsies further revealed co-expression of GREM1, JAG1 and HES1 mainly in tubular epithelial cells in patients with DN but not in normal human kidney [2]. Expression microarray data provided additional evidence for significant differential expression of ten genes involved in the Notch signalling pathway in biopsies from DN patients compared with control samples [2]. Here we assessed single-nucleotide polymorphisms (SNPs) in four of these key differentially expressed Notch pathway genes (JAG1, HES1, NOTCH3 and $A D A M 10$ ) for association with $\mathrm{DN}$ using a case-control design involving 1467 individuals with type 1 diabetes.

\section{Material and methods}

Participants Ethics approval was obtained from the appropriate university research ethics committees, and written informed consent was obtained prior to study participation. All participants were white, had type 1 diabetes diagnosed before 32 years of age and were recruited from the UK and Ireland. Patients $(n=718)$ and controls $(n=749)$ were derived from the Warren 3/UK Genetics of Kidneys in Diabetes (GoKinD) and all-Ireland collections [7]. Case definition was based on the development of persistent proteinuria $(>0.5 \mathrm{~g}$ protein $/ 24 \mathrm{~h})$ at least 10 years after diagnosis of type 1 diabetes, hypertension (blood pressure $>135 / 85 \mathrm{mmHg}$ or treatment with antihypertensive agents) and associated diabetic retinopathy. Controls were individuals with type 1 diabetes for at least 15 years with normal urinary albumin excretion rates, demonstrated no evidence of microalbuminuria on repeated testing and were not prescribed antihypertensive treatment. This avoided possible misclassification of a diabetic individual as a control phenotype when the use of antihypertensive treatment may have reduced urinary albumin excretion into the normal range. Individuals with microalbuminuria were excluded from both groups.

SNP selection and genotyping Tag SNPs for each gene investigated were selected from Phase II, release 24 HapMap (http://www.hapmap.org) CEPH data (Utah residents with ancestry in northern and western Europe; CEU) using Haploview to visualise linkage disequilibrium and haplotype blocks between variants (version 4.1; http:// www.broadinstitute.org/haploview). Tag SNPs were selected using a pairwise tagging approach where $r^{2}$ exceeded 0.8 for all downloaded SNPs with minimum minor allele frequency $(\mathrm{MAF})>5 \%$ and no deviation from HardyWeinberg equilibrium (HWE) at $p<0.001$. Additional potentially functional SNPs were identified using the Ensembl genome browser (www.ensembl.org) and SNP@Promoter [8]. Genotyping was performed by MassARRAY iPLEX (Sequenom, San Diego, CA, USA) or Taqman 5' nuclease (Applied Biosystems, Foster City, CA, USA) assays according to the manufacturer's instructions.

Quality filters for exclusion of SNPs included deviation from HWE $(p<0.001)$ and call rates below 95\% and samples with missingness $\geq 10 \%$. Additional quality measures included blind duplicates and father/mother/offspring trios on plates, allocation of patients and controls in equal proportions on each plate, independent scoring of genotypes by two individuals and resequencing of randomly selected DNAs to validate genotypes.

Statistical analysis Clinical characteristics of patients and controls were compared using the z-test for large independent samples and the $\chi^{2}$ test. Association analyses were performed using PLINK (version 1.07; http://pngu.mgh. harvard.edu/ purcell/PLINK/) [9]. Initially a $\chi^{2}$ test for trend ( $1 d f)$ was used with stratification by collection centre. Logistic regression analysis was then performed on each SNP with terms for potential confounders (collection centre, sex, duration of type 1 diabetes mellitus and $\mathrm{HbA}_{1 \mathrm{c}}$ ) included in the model. The statistical significance level was set at $5 \%$ and adjustment for multiple testing was by permutation test $(n=100,000)$. Haplotype analysis was conducted where SNPs formed a haplotype block using $95 \%$ confidence based on pairwise $\mathrm{D}^{\prime}$. Incorporating a Bonferroni correction for 50 comparisons, this collection has $90 \%$ power to identify an allele with an odds ratio of 
Table 1 Clinical characteristics of patients and controls

\begin{tabular}{|c|c|c|}
\hline Characteristic & $\begin{array}{l}\text { Patients } \\
(n=668)\end{array}$ & $\begin{array}{l}\text { Controls } \\
(n=703)\end{array}$ \\
\hline Male, $n(\%)^{* * *}$ & $386(57.8)$ & $299(42.5)$ \\
\hline Age at diagnosis of type 1 diabetes mellitus (years) ${ }^{*}$ & $14.8 \pm 7.7$ & $15.6 \pm 7.9$ \\
\hline Duration of type 1 diabetes mellitus (years) ${ }^{\mathrm{a} * * *}$ & $33.2 \pm 9.3$ & $27.9 \pm 9.0$ \\
\hline $\mathrm{HbA}_{1 \mathrm{c}}(\%)^{\mathrm{b} * * *}$ & $9.0 \pm 1.9$ & $8.7 \pm 1.6$ \\
\hline Systolic blood pressure $(\mathrm{mmHg})^{\mathrm{b} * * *}$ & $145.2 \pm 20.8$ & $125.3 \pm 14.5$ \\
\hline Diastolic blood pressure $(\mathrm{mmHg})^{\mathrm{b} * * *}$ & $81.8 \pm 11.1$ & $75.6 \pm 7.7$ \\
\hline Body mass index $\left(\mathrm{kg} / \mathrm{m}^{2}\right)$ & $26.2 \pm 4.7$ & $26.2 \pm 4.2$ \\
\hline Serum cholesterol $(\mathrm{mmol} / \mathrm{l})^{*}$ & $5.35 \pm 1.23$ & $5.09 \pm 0.92$ \\
\hline Serum creatinine $(\mu \mathrm{mol} / 1)$, median (interquartile range) ${ }^{\mathfrak{C} * * *}$ & $127(99-179)$ & $92(78-105)$ \\
\hline Glomerular filtration rate $\left(\mathrm{ml} \mathrm{min}^{-1} 1.73 \mathrm{~m}^{-2}\right)$, median (interquartile range) $)^{\mathrm{d}, \mathrm{c}_{* * *}}$ & $49(35-68)$ & $70(59-86)$ \\
\hline
\end{tabular}

Unless otherwise stated, values are mean \pm SD

${ }^{a}$ Calculated from the dates of diagnosis and recruitment

${ }^{\mathrm{b}}$ Average of the three most recent values prior to recruitment

${ }^{\mathrm{c}}$ Excludes participants receiving renal replacement therapy (dialysis or transplant)

${ }^{\mathrm{d}}$ Estimated from serum creatinine, age and sex using the MDRD equation (www.renal.org/eGFRcalc/)

${ }^{*} p<0.05, * * * p<0.001$ for patients vs controls

$1.71,1.52,1.45$ and 1.43 whose frequency in controls is 10 , 20,30 and $40 \%$, respectively.

\section{Results}

Table 1 lists the clinical characteristics of the patients $(n=668)$ and controls $(n=703)$ genotyped in this study. Unsurprisingly, there were more males, higher mean $\mathrm{HbA}_{1 \mathrm{c}}$ and blood pressure values (despite the use of antihypertensive treatment) in the patient group compared with the control group. Approximately one-third (30.6\%) of patients had end-stage renal disease (ESRD). Subgroup analyses on the basis of ESRD status offered no improvement in terms of disease association with ESRD compared with those calculated for $\mathrm{DN}$.

We excluded 96 samples (50 patients and 46 controls) from the analysis with missingness $\geq 10 \%$. A total of 38 SNPs were genotyped using MassARRAY iPLEX technology, and four SNPs (rs7271215, rs60400060 and rs6040068 in $J A G 1$, and rs8043406 in $A D A M 10$ ) were genotyped by Taqman $5^{\prime}$ nuclease assay in 668 patients and 703 controls (see Electronic supplementary material [ESM] Table 1). The average call rate for all SNPs analysed was 99.25\%. The genotype distribution for each SNP did not

Table 2 Minor allele frequencies (MAF) and allele counts in patients and controls for SNPs with a $p$ value $<0.05$

\begin{tabular}{|c|c|c|c|c|c|c|c|c|c|c|}
\hline SNP & $\begin{array}{l}\text { Gene } \\
\text { symbol }\end{array}$ & Alleles ${ }^{\mathrm{a}}$ & $\begin{array}{l}\text { Patient } \\
\text { count }\end{array}$ & $\begin{array}{l}\text { Patient } \\
\text { MAF }\end{array}$ & $\begin{array}{l}\text { Control } \\
\text { count }\end{array}$ & $\begin{array}{l}\text { Control } \\
\text { MAF }\end{array}$ & $p$ value & OR & $95 \% \mathrm{CI}$ & $\begin{array}{l}\text { Adjusted } \\
p \text { value }\end{array}$ \\
\hline rs8708 & $J A G 1$ & $\mathrm{~A} / \mathrm{G}$ & $254 / 794$ & 0.40 & $120 / 763$ & 0.46 & 0.04 & 0.87 & $0.73-1.04$ & 0.97 \\
\hline rs11699674 & $J A G 1$ & $\mathrm{G} / \mathrm{C}$ & $94 / 1242$ & 0.07 & $121 / 1281$ & 0.09 & 0.02 & 0.71 & $0.50-1.00$ & 0.75 \\
\hline rs1548555 & NOTCH3 & $\mathrm{A} / \mathrm{G}$ & $161 / 1173$ & 0.12 & $140 / 1266$ & 0.10 & 0.05 & 1.40 & $1.05-1.86$ & 0.47 \\
\hline rs10423702 & NOTCH3 & $\mathrm{T} / \mathrm{C}$ & $195 / 1139$ & 0.15 & $162 / 1242$ & 0.12 & 0.04 & 1.45 & $1.11-1.90$ & 0.19 \\
\hline rs8027998 & $A D A M 10$ & $\mathrm{G} / \mathrm{A}$ & $201 / 1127$ & 0.15 & $230 / 1174$ & 0.16 & 0.05 & 0.77 & $0.60-0.98$ & 0.62 \\
\hline rs2054096 & $A D A M 10$ & $\mathrm{~A} / \mathrm{T}$ & $384 / 950$ & 0.29 & $352 / 1050$ & 0.25 & 0.01 & 1.31 & $1.07-1.59$ & 0.20 \\
\hline
\end{tabular}

$p$ values were calculated using a $\chi^{2}$ test for trend $(1 d f)$ with stratification by centre

Odds ratios and $95 \%$ confidence intervals are for the first-mentioned allele and are adjusted for centre, sex, duration of disease and $\mathrm{HbA}_{1 \mathrm{c}}$ level

${ }^{\mathrm{a}}$ The minor allele is presented first, followed by the major allele

${ }^{\mathrm{b}}$ Associations were no longer significant after adjustment for multiple testing 
deviate significantly from HWE in patients and controls. No duplicate or Mendelian inconsistencies were observed.

Six SNPs were significant before adjustment for multiple testing: rs8708 $(p=0.04)$ and rs11699674 $(p=0.02)$ in $J A G 1$, rs10423702 $(p=0.04)$ and $\mathrm{rs} 1548555(p=0.05)$ in NOTCH3 and $\operatorname{rs} 2054096(p=0.01)$ and $\operatorname{rs} 8027998(p=0.05)$ in ADAM10 (Table 2). Logistic regression analyses with adjustment for collection centre, sex, duration of disease and $\mathrm{HbA}_{1 \mathrm{c}}$ showed a significant association for five of these six SNPs (with the exception of rs8708) but these associations were no longer significant after correction for multiple testing. Haplotype comparisons revealed no significant findings. Subgroup analysis based on median duration of diabetes $(<30$ years and $\geq 30$ years $)$ also revealed no significant associations with $\mathrm{DN}$.

\section{Discussion}

To our knowledge this is the first genetic association study to specifically assess common SNPs in the Notch pathway genes JAG1, HES1, NOTCH3 and ADAM10 for DN. The Notch pathway is an evolutionarily conserved cellsignalling mechanism essential in glomerular development and has been reported to interact with the TGF $\beta$ pathway in glomerular podocytes during disease conditions [3]. In vitro studies have identified Jagged 1 as a direct transcriptional target of TGF $\beta 1$, with increased expression of Jagged1 likely to induce Notch cleavage and activation [3]. It is noteworthy that we recently demonstrated an association between DN and an SNP (rs1129456) in GREM1 [7]. Although we did not find an association with DN that survived adjustment for multiple testing for any of the 42 SNPs assessed in this study, some SNPs were significant before adjustment, notably in JAG1, NOTCH3 and $A D$ AM10. Morrisey and colleagues [1] reported TGF $\beta 1$ mediated upregulation of Jagged1 in kidney tubules in a mouse model of renal fibrosis. Furthermore, as the Notch signalling pathway is involved in determining cell fate during development and tissue maintenance and repair in adult organs, manipulation of Notch in animal models indicates potential as a novel therapeutic approach for the treatment of chronic kidney disease [1]. Murea et al. [10] reported Notch pathway activation as a common mechanism involved in the pathophysiology of a wide range of acquired renal diseases associated with albuminuria, glomerulosclerosis and tubulointerstitial fibrosis. In addition, recently identified regulation of vascular endothelial growth factor expression by the Notch pathway has been proposed to play a key role in diabetic podocytes as they drive the development of DN [11]. Lin et al. [11] also proposed that Notch pathway modulation may offer a novel therapeutic intervention for the prevention of $\mathrm{DN}$.
Our findings may be explained by the fact that these genes are not implicated in DN. Alternatively, the effect size of SNPs investigated in this study may be small, necessitating the use of much larger sample sizes to detect them. Since our study focused only on common variants, untyped rare variants within these genes could still be associated with DN. Other factors, such as microRNAs (RNAs that regulate gene expression by binding to the $3^{\prime}$ untranslated regions of specific mRNAs) and DNA methylation, that may regulate the function of these genes, could also be implicated in DN.

In conclusion, our data suggest that common variants in the JAG1, HES1, NOTCH3 and ADAM10 genes are not strongly associated with DN in type 1 diabetes. Further work is required to investigate the potential role of rare variants and epigenetic factors within these genes and others involved in this important signal transduction pathway to determine their role in $\mathrm{DN}$.

Acknowledgements D. Kavanagh is supported by a Northern Ireland Kidney Research Fund (NIKRF) PhD studentship. We thank the NIKRF and Northern Ireland Research and Development Office for supporting this work. We also thank D. Sadlier, University College Dublin, for providing DNA samples from patients and controls from the Republic of Ireland. The Warren 3/UK GoKinD Study Group was jointly funded by Diabetes UK and the Juvenile Diabetes Research Foundation and includes the following individuals: Belfast: A. P. Maxwell, A. J. McKnight, D. A. Savage; Edinburgh: J. Walker; London: S. Thomas, G. C. Viberti; Manchester: A. J. M. Boulton; Newcastle: S. Marshall; Plymouth: A. G. Demaine, B. A. Millward; Swansea: S. C. Bain.

Duality of interest The authors declare that there is no duality of interest associated with this manuscript.

\section{References}

1. Morrisey J, Guo G, Moridaira K et al (2002) Transforming growth factor-beta induces renal epithelial jagged-1 expression in fibrotic disease. J Am Soc Nephrol 13:1499-1508

2. Walsh DW, Roxburgh SA, McGettigan P et al (2008) Coregulation of gremlin and notch signalling in diabetic nephropathy. Biochim Biophys Acta 1782:10-21

3. Niranjan T, Bielesz B, Gruenwald A et al (2008) The notch pathway in podocytes plays a role in the development of glomerular disease. Nat Med 14:290-298

4. Bray SJ (2006) Notch signalling: a simple pathway becomes complex. Nat Rev Mol Cell Biol 7:678-689

5. Hills CE, Squires PE (2010) TGF-beta1-induced epithelial-tomesenchymal transition and therapeutic intervention in diabetic nephropathy. Am J Nephrol 3:68-74

6. Dolan V, Murphy M, Sadlier D et al (2005) Expression of gremlin, a bone morphogenetic protein antagonist, in human diabetic nephropathy. Am J Kidney Dis 45:1034-1039

7. McKnight AJ, Patterson CC, Pettigrew KA et al (2010) A GREM1 gene variant associates with diabetic nephropathy. J Am Soc Nephrol 21:773-781 
8. Kim BC, Kim WY, Park D et al (2008) SNP@Promoter: a database of human SNPs (single nucleotide polymorphisms) within the putative promoter regions. BMC Bioinformatics 9(Suppl 1):S2

9. Purcell S, Neale B, Todd-Brown K et al (2007) PLINK: a toolset for whole-genome association and population-based linkage analysis. Am J Hum Genet 81:559-575
10. Murea M, Park JK, Sharma S et al (2010) Expression of Notch pathway proteins correlates with albuminuria, glomerulosclerosis, and renal function. Kidney Int 78:514-522

11. Lin CL, Wang FS, Hsu YC et al (2010) Modulation of notch-1 signaling alleviates vascular endothelial growth factor-mediated diabetic nephropathy. Diabetes 59:1915-1925 